\title{
Correction to: Knockdown of miR-155 protects microglia against LPS-induced inflammatory injury via targeting RACK1: a novel research for intracranial infection
}

Haiyan Yin ${ }^{1,2+}$, Shuwen Song ${ }^{3+}$ and Xudong Pan ${ }^{4^{*}}$

\section{Correction}

Upon publication of the original article [1] it was highlighted by the authors that the first affiliation of author Haiyan Yin was incorrectly cited in duplicate as "Department of Neurology, Affiliated Hospital of Jining Medical University, Jining, 272000, China". This should instead read "Qingdao University, Qingdao, 266,071, China". This error in the author details has since been formally noted in this correction article.

\footnotetext{
Author details

'Qingdao University, Qingdao 266071, China. 'Department of Neurology, Affiliated Hospital of Jining Medical University, Jining 272000, China. ${ }^{3}$ Department of Infectious Diseases, Jining No.1 People's Hospital, Jining 272000, China. ${ }^{4}$ Department of Neurology, The Affiliated Hospital of Qingdao University, Qingdao 266000, China.
}

Published online: 03 April 2018

\section{Reference}

1. Yin $\mathrm{H}$, Song S, Pan X. Knockdown of miR-155 protects microglia against LPS-induced inflammatory injury via targeting RACK1: a novel research for intracranial infection. J Inflamm. 2017;14(1):17.

\footnotetext{
* Correspondence: panxudong012@126.com

${ }^{\dagger}$ Equal contributors

${ }^{4}$ Department of Neurology, The Affiliated Hospital of Qingdao University,

Qingdao 266000, China

Full list of author information is available at the end of the article
} 